\title{
The Process of Multi-agent Participation in Value Co-creation under Crowdsourcing Innovation Mode Qiaoyi Cao
}

School of Economics \& Management, Jiangsu University of Science and Technology, Zhenjiang, 212003,China

2206244665@qq.com

Qiaoyi Cao

Keywords: Crowdsourcing innovation mode, Multi-agent participation, Value co-creation.

Abstract. The innovation of based on crowdsourcing platform provides innovative solutions for enterprises. Many scholars have done a lot of research on willingness and mode of innovation on crowdsourcing platform, but the papers regarding value co-creation in the crowdsourcing process are relatively less. Therefore, this paper explores the specific process of multi-agent participation in value creation under crowdsourcing innovation mode, through sorting out and summarizing of a large number of relevant literature.

\section{众包创新模式下多主体参与价值共创的过程}

\author{
曹乔怡 \\ 江苏科技大学经济管理学院, 镇江, 212003 \\ 2206244665@qq.com
}

曹乔怡

关键词：众包创新模式; 多主体参与;价值共创

中文摘要. 平台型的众包创新为企业提供了创新需求的解决方案, 许多学者在参与意愿、创新 模式等方面对众包创新做了大量的研究, 但是结合众包创新与价值共创的研究相对较少。基 于此, 本文通过对大量相关文献的梳理和总结, 探究众包创新模式下多主体参与价值共创的 具体过程。

1. 引言

创新是企业获得和维持竞争优势的关键要素。随着互联网和信息技术的高速发展, 企业 创新由完全封闭式创新转变为开放式创新, 创新范围已不局限于企业内部, 而是向着更为广 泛的社会范围拓展。在此背景下, 众包创新模式应运而生。2006 年, HOWE 在美国《连线》 上首次提出了众包的概念，他认为众包是一种新的商业模式，即企业利用互联网或通过第三 方众包平台将创造企业核心价值的重任置于外部大众身上 ${ }^{[1]}$ 。近年来，国内外许多知名企业 采用众包创新模式纷纷取得了商业成功。菲亚特汽车公司通过众包创新模式成功设计出 Fiat500 车型, 竞赛期间共收到 Fiat500 车型设计方案 17 万个, 同时还有铬保险杜、排气管造

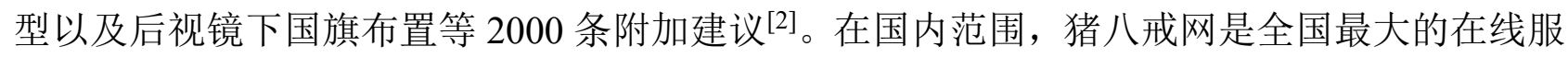


务交易平台, 其服务交易品类涵盖创意设计、网站建设、网络营销、文案策划、生活服务等 多个行业。为企业、公共机构和个人提供定制化的解决方案。Zhang Peng 等通过总结众包实 践案例, 根据众包运营商和参与者关系结构两维度, 把众包模式分为四类: 集中建议型 (例如 星巴克咖啡 Star-bucks Coffee)、众包创新平台、istockphoto 类平台、Threadless 在线 $\mathrm{T}$ 恤设 计类平台 ${ }^{[3]}$ 。在众包创新过程中, 不同参与者出于经济因素、心理因素等方面的考虑, 选择 相互合作, 通过资源整合、知识创新等方式, 从而产出价值。可见, 众包创新过程也是发包 方、接包方与众包平台三方共同创造价值的过程，即价值共创的过程。

价值共创是新兴的研究课题, 价值共创思想最早是由 Norman 和 Ramirez 在 1993 年提出, 他们认为价值共创系统是成功企业战略分析的关注点, 这个系统包括了供应商、合作方、顾 客等多方经济行为者, 系统所有成员共同生产价值 ${ }^{[4]}$ 。2000 年, Prahalad 和 Ramaswamy 首次 正式提出了价值共创的概念, 指出共同创造消费体验是消费者与企业价值共创的核心, 价值 网络成员间的互动是实现价值共创的基本方式 ${ }^{[5]}$ 。此后, 众多国内外学者对价值共创进行了 不同方向的研究。Stephen L. Vargo 等认为价值共创是指参与交换的各个服务系统共同创造价 值, 从而促进产品的创新和改进 ${ }^{[6]}$ 。从资源维度来讲, 钱轶由认为企业首先提出价值主张, 然后作为资源拥有者投入硬件软件等资源，同时有参与意向的顾客作为资源拥有者也投入到 价值共创的系统中, 双方通过持续的对话、获取等来实现价值共创[7]。价值共创是多方参与 者共同投入资源, 进行整合和创新, 产出价值的过程, 这也与众包创新中价值的实现过程相 一致。

但是，目前关于众包创新过程中的价值共同创造问题的研究相对较少，本文将众包创新 和价值共创结合起来, 研究众包创新模式下多主体参与价值共创的过程问题。

\section{2. 众包创新的一般运行过程}

如图 1 所示, 平台型众包创新模式是由发包方、接包方及众包平台三方主体组成, 其核 心目标是解决发包方的创新任务, 继而实现三方的共赢。在运作机制上, 首先由发包方在分 析自身制度、能力及学习等方面因素后, 发现需要解决的创新问题, 明确创新目标, 进而对 创新任务进行设计; 然后, 发包方通过众包平台发布竞赛任务, 接包方选择任务, 投入资源 与发包方进行信息交流, 并提交解决方案; 最后, 由发包方䇥选并确定最佳解答方案, 众包 平台将任务奖金支付给提供该方案的解答者，并收取相关费用。

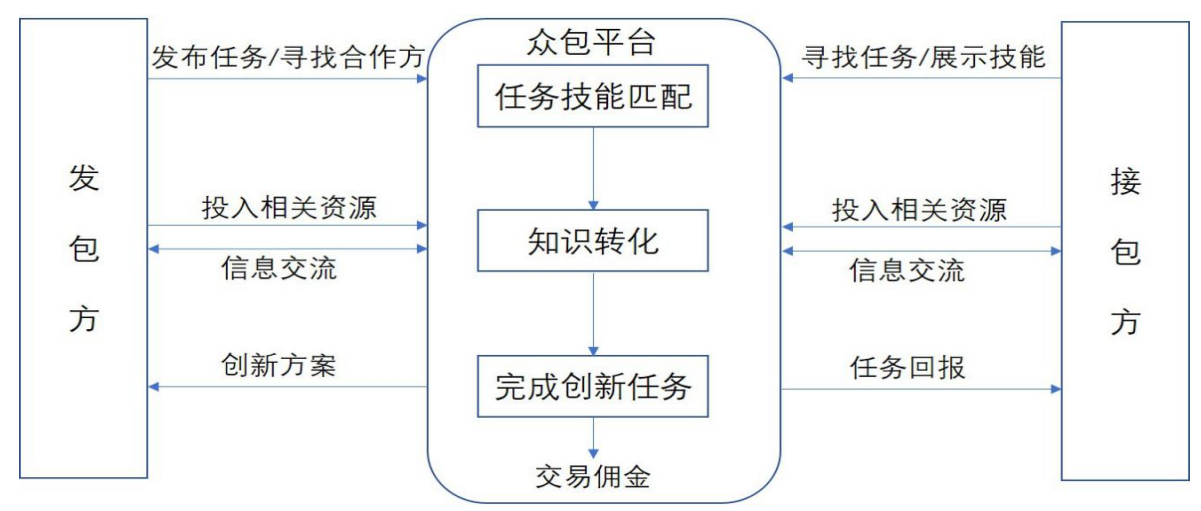

图 1 众包创新的一般流程图

\section{3. 众包创新模式下多主体参与价值共创的过程}

\section{1 众包创新模式下价值共创的关键活动}

在顾客与企业的二元结构中，两方通过组织过程资源、定义需求、设计和产生方案、执 行方案和管理价值冲突五个环节, 定义了顾客和企业在价值共创过程中的不同角色, 并最终 
产生了使用价值 ${ }^{[8]}$ 。而在三方参与的众包创新环境下, 发包方的部分角色和行为由众包平台 完成, 相应的价值共创过程也发生了变化。此时的价值共创发生在三方交叉范围的互动之中, 互动行为由人际互动、信息反馈等维度构成 ${ }^{[9]}$, 相互认可、建立情感等互动行为构成了价值 共创活动的基本要素 [10]。Mina Tajvidi 等人通过实证分析得出, 顾客与顾客间互动以及顾客与 企业间互动正向影响了社会支持，社会支持进一步正向影响顾客对企业品牌价值共创的参与 意愿 ${ }^{[11]}$ 。基于此, 本文认为关系互动是众包创新模式下多主体参与价值共创的一项关键活动。

同时，价值共创过程需要知识等资源的分享作为基础，且价值共创的前提就是资源的互 补性。江积海和李琴提出价值共创的参与者通过资源共享、知识创新以及资源互动提升, 促 进了价值共创的实现 ${ }^{[12]}$ 。张婧和何勇以服务主导逻辑为导向, 通过实证研究发现提高资源互 动能力能有效促进价值共创活动, 进而提升顾客感知价值 ${ }^{[13]}$ 。周文辉运用扎根理论编码的案 例研究方法对知识服务、价值共创和创新绩效的关系进行了分析和论证, 发现知识服务的内 容和价值共创的过程相互作用, 共同促进创新绩效的产生 ${ }^{[14]}$ 。在众包创新环境中, 知识的来 源更多集中在接包方和发包方, 两方的知识分享对于价值共创活动起到了重要作用。而作为 众包平台, 一方面要加强技术、人员等资源投入来增强互动效果, 另一方面也要通过完善交 易及激励制度、制作指引工具等为知识分享创造便利条件, 协助接包方和发包方实现价值共 创的过程。因此, 本文认为知识分享是众包创新模式下多主体参与价值共创的另一项关键活 动。

\section{2 众包创新模式下多主体参与价值共创的过程}

众包创新的参与主体有接包方、发包方、众包平台, 三方的结构是非正式的组织, 由协 议和制度等作为约束条件, 投入知识等资源, 产出创新任务的解决方案。而在价值共创中, 企业、顾客、供应商等利益相关者同时组成了非正式的联合体, 共享统一的价值观念, 通过 分享信息、知识等资源, 产生创新思路或技术方案。可见, 众包创新的过程同时也是价值共 创的过程，即众包创新的本质就是价值共创。

众包创新的参与方本身就是生产者与消费者的关系，三方共同遵守着一定的规则和协议。 发包方通过众包平台发布创新任务, 寻找合作方, 同时提出价值主张; 接包方通过平台寻找 任务, 接受价值主张; 众包平台将任务需求与知识技能相匹配, 促进价值认同。而后, 接包 方和发包方分别投入知识、信息等资源, 进行信息交流、知识分享与关系互动; 众包平台则 通过制度安排、过程介入、关系协调等行为, 与接包方和发包方共同创造价值。最后, 三方 通过价值共创活动解决了创新任务, 对产出价值进行了分配, 从而获取了各自的价值。对于 发包方而言, 其参与众包创新活动的目的主要是为了获取创新方案和思路, 解决自身发展难 题, 从而使自身在激烈的竞争环境中保持优势甚至超越竞争对手, 立于不败之地。对于接包 方而言, 其参与众包创新活动的动机有许多, 从外部动机来看, 主要是为了获取经济报酬, 获得经济优势, 这也是最直接的参与动机; 从内部动机来看, 接包方参与众包创新活动是为 了满足某种心理需求, 从而获得心理上的满足感。对于平台而言, 其不仅是作为其他两方进 行知识分享和资源互动进行价值创造活动的中介, 平台本身也需要建立和完善相应的规则制 度, 提供更合理的服务, 促进资源转换和价值实现, 继而获得相应的佣金报酬, 增强其他两 方对平台的信任和忠诚度，从而提高平台的知名度和影响力。

根据以上的分析，本文提出了众包创新模式下多主体参与价值共创的过程图。 


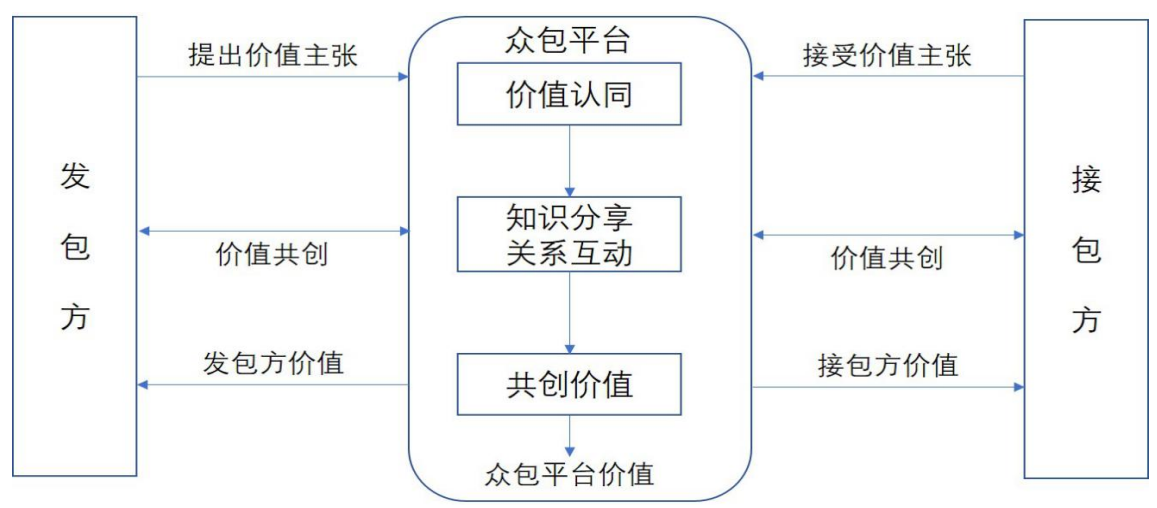

图 2 众包创新模式下多主体参与价值共创的过程

\section{4. 结束语}

众包创新模式为越来越多的企业所采用,对企业的开放性创新起到了巨大的推动作用，并 掀起了整个社会范围内的创新热潮。本文通过对众包创新与价值共创的参与主体、过程行为 及结果等方面的分析得出, 众包创新的本质即是价值共创。但是, 目前对于众包创新环境下 的价值共创问题的研究处于相对空白的状态。因此, 本文将价值共创活动与众包创新过程进 行了有机的融合, 对发包方、接包方和众包平台三方参与的众包创新过程进行分析, 提出了 众包创新模式下多主体参与价值共创的运行图。本文的研究对于众包创新与价值共创的融合 研究提供了新的思路, 同时对于三方的实践也具有一定的启示作用。但是, 本文也存在不足 之处：一是未对众包创新模式进行具体的细分, 仅以一般性的众包创新为研究背景, 对其中 的价值共创过程做了研究; 二是本文仅对众包创新模式下多主体参与价值共创的过程进行研 究, 并没有深入考虑其影响因素和产出价值; 三是缺乏实证研究, 本文是通过文献研究、逻 辑分析等定性分析法形成, 未进行定量分析。鉴于本文的不足之处, 未来的研究需要对众包 创新环境进行细分和定位, 明确具体类型或众包平台, 增强针对性。还有可以从前因-过程结果这个思路研究众包创新模式下多主体参与价值共创的实现机制问题, 使问题考虑的更加 全面。最后, 未来的研究可以通过采取定量分析方法, 以真实数据检验概念模型的有效性, 对概念模型进行完善和发展。

\section{References}

[1] Howe J. The rise of crowdsourcing[J]. Wired magazine, 2006, 14(6): 1-4.

[2] Kleemann F, Voß G G, Rieder K. Un(der)Paid Innovators: The Commercial Utilization of Consumer Work through Crowdsourcing[J]. 2008, 4(2):5-26.

[3] Zhang P, Lu R. On operating mechanism of Crowdsourcing service innovation[C]// International Conference on Service Systems and Service Management. IEEE, 2011:1-8.

[4] Normann R, Ramirez R. Designing interactive strategy[J]. Harvard Business Review(july-august, 1993.

[5] Prahalad C K, Ramaswamy V. Co-creating unique value with customers[J]. Strategy \& Leadership, 2004, 32(3):4-9.

[6] Vargo S L, Maglio P P, Akaka M A. On value and value co-creation: A service systems and service logic perspective[J]. European Management Journal, 2008, 26(3):145-152.

[7] QIAN Yiran.Research of Simulation Design Based on Product Experience[J].Art And Design, 2008(9):144-145.

[8] Aarikka-Stenroos L, Jaakkola E. Value co-creation in knowledge intensive business services: A 
dyadic perspective on the joint problem solving process[J]. Industrial Marketing Management, 2012, 41(1):15-26.

[9] BU Qing-juan, JIN Yong-sheng, LI Zhao-hui.Measurement and Verification of Customer Value Co-Creation Interactive Behaviors within Virtual Brand Community[J]. Contemporary Finance \& Economics,2016(5):76-86.

[10] Busser J A, Shulga L V, Busser J A, et al. Co-created value: Multidimensional scale and nomological network[J]. Tourism Management, 2018, 65:69-86.

[11] Tajvidi M, Wang Y, Hajili N, et al. Brand Value Co-creation in Social Commerce: The Role of Interactivity, Social Support, and Relationship Quality[J]. Computers in Human Behavior, 2017.

[12] Jiang Jihai, Li Qin. Effect of Connection Attributes of Platform-based Business Model Innovation on Value co-creation Based on Case Study of Airbnb[J]. Management Review, 2016, 28(7):252-260.

[13] Zhang Jing, He Yong, Impact of service-dominant logic orientation and resource interaction upon value co-creation[J]. Science Research Management, 2014, V35(1):115-122.

[14] Zhou Wenhui, Realization mechanism of business model innovation driving technology innovation:A multiple case grounded theory method according to software industry[J]. Studies in Science of Science, 2015, 33(4):567-573. 